Bundesgesundheitsbl 2013 · 56:1340-1341

DOI 10.1007/s00103-013-1838-7

(c) Springer-Verlag Berlin Heidelberg 2013

Mitteilungen des Arbeitskreises Blut des Bundesministeriums für Gesundheit

\title{
Befristete Rückstellung von der Blutspende bei Personen mit sexuellem Risikoverhalten
}

\author{
Stellungnahme des Arbeitskreises \\ Blut (S12) zum Beratungsergebnis \\ der Bundesärztekammer
}

Relevant sind in diesem Zusammenhang u. a die folgenden Faktoren:

- Verständlichkeit der Darstellung,

- Schaffung von Verständnis und Akzeptanz der zum Ausschluss führenden Fragen,

- Vermeidung eines Eindrucks von Diskriminierung,

- Verminderung des Einflusses anderer, nicht direkt mit der Blutspende zusammenhängender Faktoren, die die Motivationslage bei der Ausfüllung des Bogens beeinflussen können (z. B. andere Interessen, sog. „Testseeking“, Protest-Verhalten als Reaktion auf vermutete Diskriminierung etc.).

Um eine Steigerung der Blutsicherheit $\mathrm{zu}$ erreichen, ist zwingend erforderlich weitere Maßnahmen zur Erhöhung der Spenderadhärenz umzusetzen. Hierzu gehören die Reduktion von Fehlanreizen für die Spende, z. B. Wunsch nach einem HIV-Testergebnis durch ein leicht zugängliches, anonymes und kostenloses Testangebot, Transparenz und Verständlichkeit der Spenderauswahlkriterien und aktive Kommunikation mit Hauptbetroffenengruppen.
Ergänzend stellt der AK Blut fest:

1. Es ist nicht Aufgabe des AK Blut, Rechtsfragen im Zusammenhang mit der Auslegung von EU-Richtlinien zu klären.

2. Der AK Blut kann keine Prognose hinsichtlich der Abnahme von Plasma zur Fraktionierung durch pharmazeutische Unternehmer vornehmen. Einigen Spendeeinrichtungen liegen jedoch schriftliche Stellungnahmen der Plasmaindustrie vor, aus denen deutlich wird, dass für die Abnahme von Plasma zur Fraktionierung ein Dauerausschluss von MSM weiter gefordert wird. Daher befürwortet der AK Blut eine Änderung der Hämotherapierichtlinien, sofern die internationale Verkehrsfähigkeit von Plasma zur Fraktionierung als Ausgangsstoff für Plasmaderivate gewährleistet bleibt.

3. Es hat sich durch das umfangreiche ausgewertete empirische/epidemiologische Datenmaterial gezeigt, dass im Bereich der durch Spenderbefragung beabsichtigten Risikominimierung mehrere Aspekte von Relevanz sind. Neben den sachlichen Aspekten wie Inhalt und Differenziertheit der Fragen sind Fragen der realen Adhärenz der Spender entscheidend für die Auswirkung auf die Produktsicherheit. 
— Ob im Falle einer Änderung der Hämotherapierichtlinien ein zusätzlicher Sicherheitsgewinn durch die angestrebte verbesserte Akzeptanz der Spenderauswahlkriterien erreicht wird, bleibt aufgrund fehlender Daten offen.

Diese Hoffnung besteht, weil aus der Vielzahl von Gesprächen mit Fachkreisen und betroffenen Personengruppen der Eindruck entstand, dass allein schon präzisere Fragen und eine befristete Rückstellung die Akzeptanz des Fragebogens erhöhen könnten. Die Erfahrung in anderen (EU-)Staaten, die statt eines Dauerausschlusses bei sexuellem Risikoverhalten eine zeitlich befristete oder differenzierte Rückstellung durchführen, zeigt, dass zumindest kein zusätzliches Risiko für Transfusionsempfänger festgestellt wurde (http://www.edqm.eu/medias/fichiers/paphts_11_28_2r_european_committee_partial_agreeme.pdf).

Die in den Hämotherapierichtlinien niedergelegten Spenderauswahlkriterien beruhen letztlich auf harmonisiertem EU-Recht (EU-Richtlinie 2004/33/EC), zu dessen Umsetzung die Mitgliedstaaten verpflichtet sind.

Der AK Blut bittet deshalb das BMG, die nationale Umstellung des Fragebogens auf ihre EU-rechtliche Vertretbarkeit zu prüfen und auf eine EU-einheitliche Rechtspraxis hinzuwirken.

Ob hierzu eine förmliche Änderung der EU-Richtlinie erforderlich ist oder ob eine auf EU-Ebene konsentierte Auslegung der geltenden EU-Richtlinie ausreichend ist, entzieht sich der Beurteilung des AK Blut.

Für den Arbeitskreis Blut

Prof. Dr. R. Burger,

Vorsitzender

Dr. R. Offergeld,

Geschäftsführerin 